\title{
Rapidly IPv6 multimedia management schemes based LTE-A wireless networks
}

\author{
Abeer Twakol Khalil ${ }^{1}$, A.I. Abdel-Fatah ${ }^{2}$, Hesham Ali Sakr ${ }^{3}$ \\ ${ }^{1}$ Electrical Engineering Department, Faculty of Engineering, Banha University, Egypt \\ ${ }^{2}$ Electronics and Communications Engineering Department, Faculty of Engineering, Mansoura University, Egypt \\ ${ }^{3}$ Institute of Public Administration, Electronics and Computing Department, Saudi Arabia
}

\begin{abstract}
Article Info
ABSTRACT

Article history:

Received Jan 15, 2019

Revised Mar 23, 2019

Accepted Apr 19, 2019

\section{Keywords:}

GMPLS

IPv6

LTE-A

Mobility

OPNET 17.5

Scalability

Traffic Engineering

Ensuring the best quality of smart multimedia services becomes an essential goal for modern enterprises so there is always a need for effective IP mobility smart management schemes in order to fulfill the following two main functions: (I) interconnecting the moving terminals around the extended indoor smart services. In addition, (II) providing session continuity for instant data transfer in real-time and multimedia applications with negligible latency, efficient bandwidth utilization, and improved reliability. In this context, it found out that the Generalized Multi-Protocol Label Switching (GMPLS) over LTE-A network that offers many advanced services for large numbers of users with higher bandwidths, better spectrum efficiency, and lower latency. In GMPLS, there is an elimination of the routing searches and choice of routing protocols on every core LTE-A router also it provides the architecture simplicity and increases the scalability. A comparative assessment of three types of IPv6 mobility management schemes over the LTE-A provided by using various types of multimedia. By using OPNET Simulator 17.5, In accordance with these schemes, it was proven that the IPv6-GMPLS scheme is the best choice for the system's operation, in comparison to the IPv6-MPLS and Mobile IPv6 for all multimedia offerings and on the overall network performance.
\end{abstract}

Copyright $\odot 2019$ Institute of Advanced Engineering and Science. All rights reserved.

\section{Corresponding Author:}

Hesham Ali Sakr,

Department of Electronic and Computer Engineering,

Institute of Public Administration- Abha- Saudi Arabia.

Email: Hesham_sakr2010@yahoo.com

\section{INTRODUCTION}

Nowadays, by the use of advanced smartphones a serious issue could be noticed clearly with the currently using of Long Term Evolution Advanced (LTE-A) networking approaches that are very limited with regard to high mobility, increased offering of larger bandwidth and higher acceptable values demand of multimedia quality of service (QoS). In addition, the wide spread of new advanced devices and services are making mobile networking even more complex also causes network congestion problems. For example, the emerging end-user applications such as video streaming, voice and web browsing have to fulfill certain QoS requirements, such as low latency and guarantees of constant data rate. In this regard, the IP traffic management process represents a major issue in mobile communication [1]; thus, the IP management scheme has a major role regarding the service performance and the overall network performance. Furthermore, there is no doubt that the transmission speed of wireless communications is constantly increasing with the appearance of new advanced and enabling technologies [2].

Therefore, many researchers have proposed several significant modifications that have been demanded in order to attain robust next generation LTE-A architecture. Hence, the IP mobility management 
(i.e. IP handover/handoff management) is considered to be the main concern in LTE-A architecture with regard to bringing mobility [3]4]. In this sense, Meenakshi Sundaram R. proposed a new integrated IPv6-MPLS architecture that offers IP-integration, end-to-end quality of service, security, scalability, resiliency and management enhancements for the deployment of data, voice and video services. In addition, they have established a test bed in order to test the performance of their proposed architecture [5]. Furthermore, Dr. S. Thabasu kannan and T. Vengatesh provided an unprejudiced experimental performance analysis between the two protocol stacks (IPv4 and IPv6), focusing on how it related to the performance on identical settings. They found out that the network performance does not depend only on the IP version and traffic type, but also on the choice of the OS. This experimental assessment proved beyond any doubt that the IPv6 is not yet a mature enough technology; thus, in many cases, the performance of IPv6 proved to be worse than IPv4, enduring an overhead that is much higher than its expected theoretical counterpart [6]. J. Amutha, et al,. proposed an Integrated Secure Architecture for IPv4/IPv6 Address Translation between IPv4 and IPv6 Networks, with an IPv4/IPv6-Enabled Gateway Translator (IP46EGT); and that is in order to attain MAClevel, VPN-IPSec and Certificate level security. In this regard, they evaluated the network performance and tabulated the acquired results. This newly suggested architecture provided data integrity, availability and authentication, hence providing end-to-end secure communication between the source and the destination [7]. Within the same context, Yunes Abdussalam Amgahd and Raghav Yadav provided a detailed study about the current state-of-the-art Mobile IP protocols in Mobile IP networks. First, they discussed the existing works on routing and handoff; then, they analyzed the existing security mechanisms by categorizing the security mechanisms as follows: security on route optimization and security during handoff. After that, they provided an overview for the key concept, used metrics, and the advantages and drawbacks of each mechanism [8].

Moreover, Walaa F. Elsadek and Mikhail N. Mikhail addressed the assessment of IP mobility and the difficult challenges before global mobility in real 4G LTE deployments. In this regard, the focus of researchers has always been attracted by software defined networking (SDN) with its logical centralized control plane; and that is in order to affect the decision directly during real time motion. Therefore, some SDN researchers have suggested several attractive ideas in order to enhance the mobility performance in real deployments with seamless expansion cross providers; while other researchers had fears about the performance degradation, as the controller represents a single point of failure. On the other hand, other researchers oversimplify the existing challenges in mobility, and offer non-feasible solutions [9]. A. Elakkiya and P. Selvaraj, on the other hand, introduced new SDN-based architecture for the LTE packet core network, in order to enhance the QoS management. In this context, they suggested a dynamic QoS-based IP handoff management procedure with the purpose of handling the application-centric mobility management. This QoS-based handover process is meant to ensure the required level of quality for the on-going connections in accordance with the policies enforced by the SDN controller. Although the same IP handover mechanism may not able to fulfill the various requirements of the applications or the several networking scenarios in an optimal manner, the introduction of SDN in LTE network has enabled the management of mobile networks with utmost flexibility in lower cost [10].

According to the previous studies mentioned above, this current case study will modify on the weakness points provided through the use of Mobile IPv6, Mobile IPv4 and IPv6-MPLS schemes on the core infrastructure of $4 \mathrm{G}$ networks in the previous studies by using two implemented IP traffic management schemes the IPv6-MPLS and IPv6-GMPLS based the 4.5G networks. this current paper aims to add new ideas and contributions regarding the following: (I) Throughput: Usually, a high amount of data drops during the process of multimedia streaming transmission, leading to the low quality of the received data; nonetheless, higher throughput rates could be achieved through the use of IPv6-GMPLS based LTE-A network. In addition, (II) Delay Reduction: The issue of higher delay ranges during the process of data transmission is present at all multimedia applications; nonetheless, the delay rates could be decreased under conditions of increasing the coverage and mobility, through the use of IPv6-GMPLS over LTE-A. Furthermore, (III) Increasing Coverage Area: We can solve the delay issue providing high performance, through the use of IPv6-GMPLS that enables us accessing multimedia anywhere and increasing the distance. In addition, (IV) Bandwidth: The issue of bandwidth limitations faced by multimedia during the process of transmission could be solved through the use of IPv6-GMPLS based LTE-A network.

That is to say, an improvement of the multimedia QoS parameters, as well as increasing the speed of multimedia access, with higher rates of SNR and lower rates of BER could be achieved. In this regard, this current paper suggests a new approach represented in the proposed metrics for evaluating the QoS performance of the LTE-A network; and that is by covering the IPv6-labeling mechanisms in networks beyond $5 \mathrm{G}$ that occur between the eNBs. In addition, no all previous studies addressed the impact of using the proposed schemes compared to the other traditional mobile IPv6 scheme on the multimedia and the overall network performance. That is to say, in this current paper, the researcher will provide a comparative study between the MiPV6 scheme, IPv6-MPLS scheme and the IPv6-GMPLS scheme through the 
measurement cases. Hence, the main objective of this current research is to analyze, characterize and differentiate between the performance of the three schemes based on the LTE-A network, resulting in the decrease of the packet loss during the process of transmission, as well as the improvement of the overall network performance through the use of IPv6-GMPLS scheme. The rest of research is: section 2 which discusses the research methods used in this paper and the QoS matrics used in our framework. Then section 3 which illustrates the simulations results and the general discussion of results. Finally, section 4 which includes the conclusion of research and future remarks.

\section{RESEARCH METHOD}

\subsection{Mobile IPv6}

MIPv6 permits path optimization that allows the central node to perceive the location of the mobile nodes after sending its first packet; i.e. whilst the central Node $(\mathrm{CN})$ transmits the primary packet to the mobile Node (MN). The mobile IPv6 packet as shown in Figure 1 could be transmitted to the home agent of the $\mathrm{MN}$ that forwards it to the overseas agent serving the MN at this actual time [11-17].

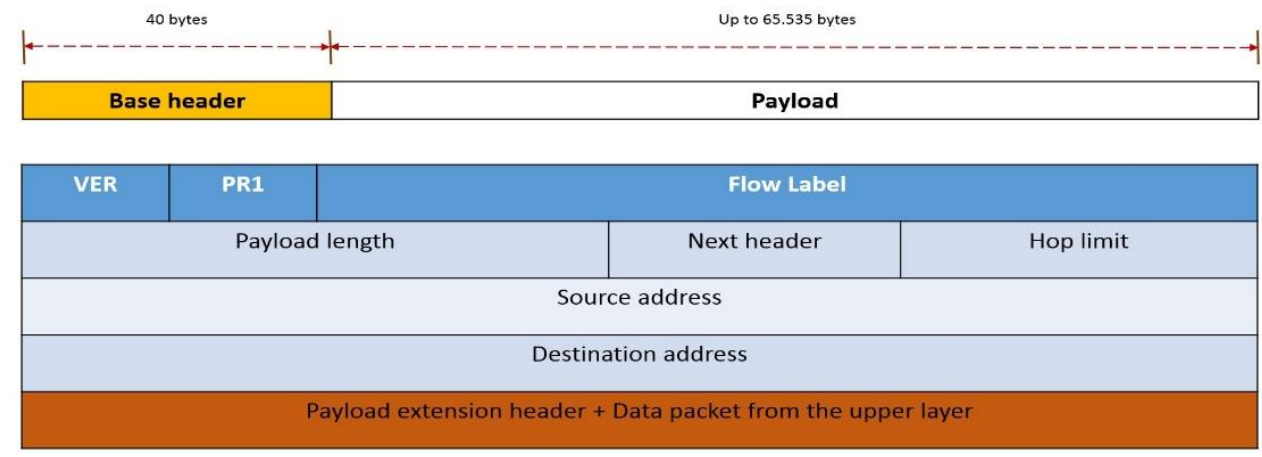

Figure 1. Mobile IPv6 header

\subsection{MPLS}

Not like the IP protocol, MPLS reduces the quantity of routing searches and gets rid of the need to have a specific routing protocol on every router. With the aid of assigning a label to every packet, it's far feasible to keep the simplicity of an structure and additionally to growth its scalability. Multi-protocol label switching (MPLS) is a generation for integrating the distinctive sorts of information including net textual content, audio (voice), video and many others. from numerous assets, e.g., Ethernet, WLANs and ATM; within the other phrases, it lets in the MPLS traces to extend the traffic handling abilities of the second layer (in line with open system Interconnection (OSI) model) [18-22], e.g., Asynhcrouns transfer mode (ATM) and frame Relay networks. Consequently, MPLS traffic generation could be very vital for multimedia service carriers and typically internet provider companies (ISPs) considering backbone or core networks have to assist an to be had high bandwidth for those usages, so the networks should be very stable, reliable and excellent quality of service (QoS) assured wherein they are able to resist conversation link or node disasters. QoS may additionally have many unique sorts of meanings in every application, as an example a network may be put off aware (for real-time usages), bit rate-aware and so forth. As illustrated in Figure 2, whilst a Label switching router (LSR) gets a packet, it performs one or extra of the subsequent movements: (I) push: provides a label. That is normally carried out with the aid of the ingress router. Additionally, (II) swapping: Replaces a label. That is normally done by means of LSRs among the ingress and egress routers. Moreover, (III) pop: eliminates a label. That is most usually done with the aid of the egress router. This diagram illustrates how an easy MPLS network works as shown in Figure 3 [23-27].

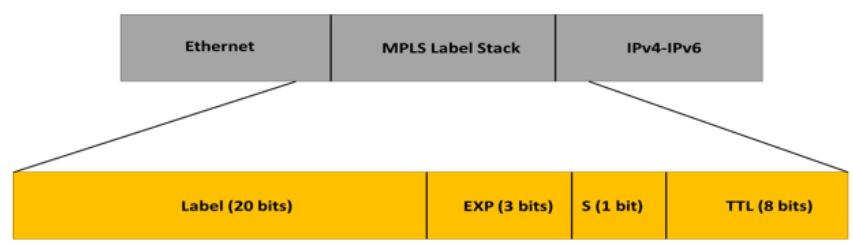

Figure 2. MPLS label components 


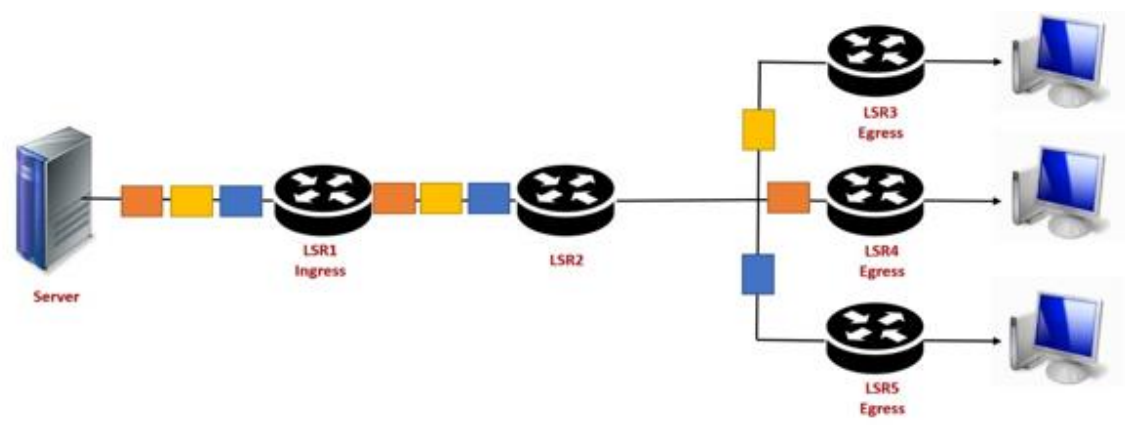

Figure 3. MPLS operation

\subsection{GMPLS}

GMPLS is conceptually just like MPLS, but rather than the use of a specific label to differentiate an LSP at every LSR, a few physical assets of the obtained data flow is used to infer which LSP it belongs to. The maximum generally used schemes are (I) the timeslot usage to become aware of the LSP, by using the time division multiplexing (TDM). Also (II) the wavelength usage to pick out the LSP, through the use of wavelength division multiplexed (WDM). Finally, (III) fiber or port on which a packet is obtained. LSPs are consequently implicitly labeled in a GMPLS network. GMPLS may be used to set up LSPs for circuit traffic (similarly to packet traffic). The usage of the TDM and WDM examples above, the LSP traffic is switched primarily based on continuous, steady assets of the information flow. The information flow is not always switched one packet at a time. This permits for a totally efficient implementation inside the data plane with 0 in keeping with-packet lookups, making GMPLS a exceptionally suitable protocol to run in excessive bandwidth networks. Beside this, GMPLS forwarding operation of the LSRs is much like the MPLS example mentioned above in Figure 3. At every LSR, the implicit label on obtained data determines the outgoing interface and the implicit label with which to transmit onwards data [28-30]. As shown in Figure 4, for MPLS, one in every of three vital signaling protocols label distribution protocal (LDP), resource reservation protocol for traffic engineering (RSVP-TE) and border gateway protocal (BGP) are used, relying at the application [31-32].

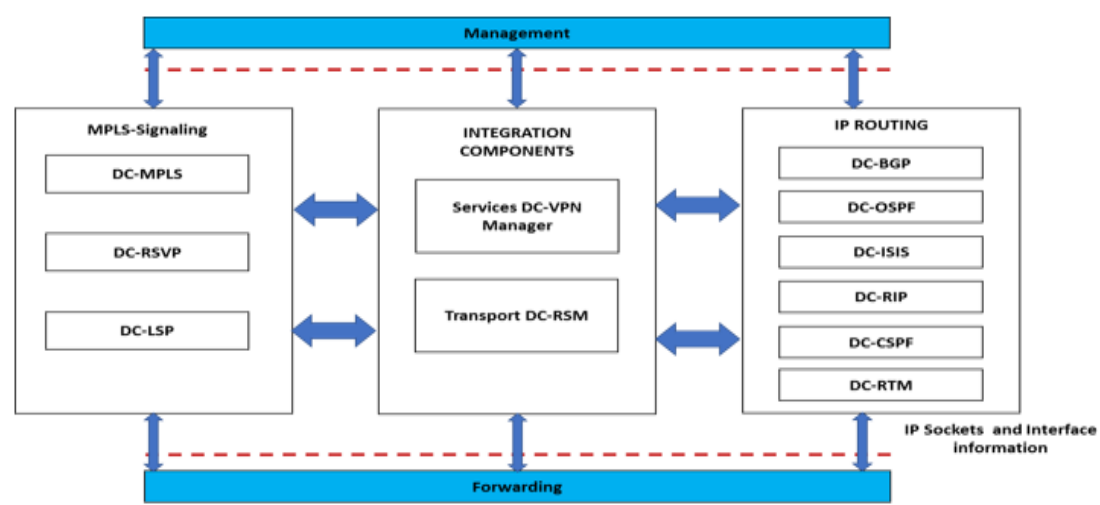

Figure 4. MPLS and GMPLS protocols

\subsection{LTE-A overview}

This section is concerned with the required basic knowledge and information, in order to fully understand the nature of the attacks on LTE-A networks. In this context, this section will provide an overview about the LTE-A network architecture as well as its security mechanism. Figure 5 illustrates the structure of the LTE-A network in detail. it is particularly consisted of a radio access network and a core network. As for the radio get right of entry to network, it's miles referred to as the E-UTRAN; as it developed from the original 3GPP UMTS terrestrial radio access network (UTRAN); consequently, the abbreviation (UMTS) refers back to the universal mobile telecommunication system. on this regard, the E-UTRAN includes multiple evolved-NodeBs (e-NodeBs), that have the identical features of the NodeBs, additionally, to many features of the Radio Network Controller within the UTRAN. Furthermore, the user equipment (UE) 
and the e-NodeB are connected via the air interface (Uu interface) [33]. Moreover, the e-NodeBs are linked to every other via the X2 interface, as they're especially involved with the subsequent features: radio resource management, IP header compression and encryption for the user information, connection to the Mobility management Entity (MME) through S1-MME interface (to be able to achieve mobility management, paging customers, and passing None access Stratum (NAS) signaling, and many others.), and connection to the Serving Gateway (SGW) with the aid of the S1-U interface. Moreover, the LTE-A additionally helps HeNBs and relay node $(\mathrm{RN})$; consequently, whilst a wide variety of HeNBs are deployed, home Base Station Gateway shall be deployed [34]. Figure 5 illustrates the structure of the LTE-advanced for the E-UTRAN. It is especially consisted of P-GW, S-GW, MME, S1-MME, eNB, HeNB, HeNB-GW and relay node as follows [35-37].

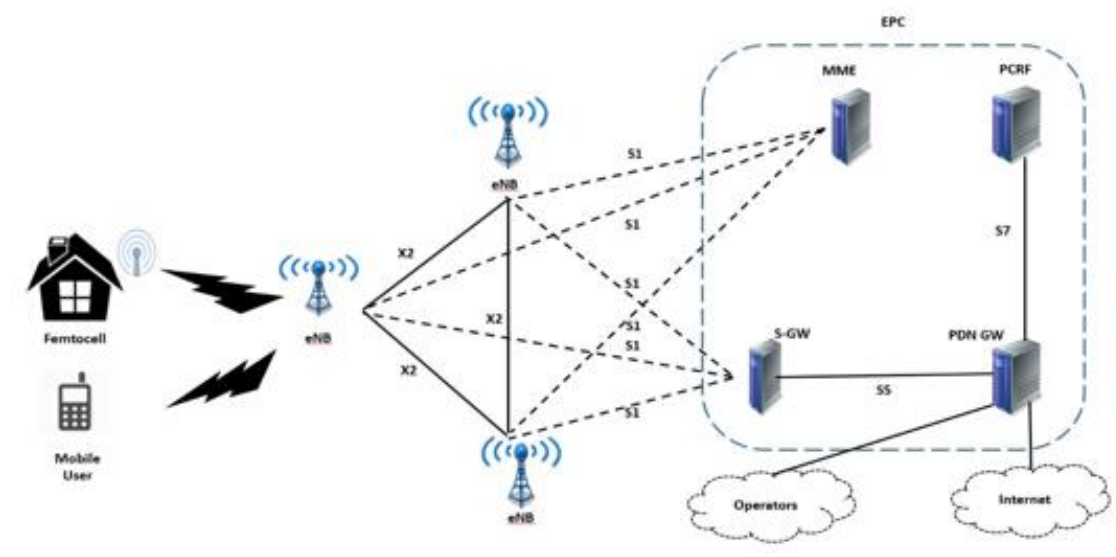

Figure 5. Architecture of the LTE-A network

\subsection{QoS Performance Metrics}

\subsubsection{End to end delay}

As illustrated in (1) and (2), the delay for one manner shall be much less than 200ms; in any other case, any E2E delay extra than $400 \mathrm{~ms}$ shall be taken into consideration as unacceptable. Certainly, this component expresses an accurate estimation, for this reason, every router has its very own dtrans, dprop, dproc [38].

$$
\begin{aligned}
& d_{E 2 E}=N\left[d_{\text {trans }}+d_{\text {prop }}+d_{\text {proc }}+d_{\text {queue }}\right] \\
& N=\text { Number of Links (Number of Routers }-1)
\end{aligned}
$$

Where: (I) $d_{\text {end-end }}$ is referring to the end-to-end delay; also (II) $d_{\text {trans }}$ is referring to the transmission delay; (III) $d_{\text {prop }}$ is referring to the propagation delay; (IV) $d_{\text {proc }}$ is referring to the processing delay; and (V) $\mathrm{d}_{\text {queue }}$ is referring to the queuing delay.

\subsubsection{Packet delay variation}

PDV should be less than 60ms; therefore, there are a subsequent 4 parameters which recognized so to calculate PDV: (I) Timestamp of the second transmitted packet; (II) timestamp of first transmitted packet; (III) timestamp of the second one arriving packet; and (IV) timestamp of the primary arriving packet. That is to mention, if the packet latency is constant value, PDV will equal zero because the difference in latency does no longer change from packet to packet, as illustrated in (3) [39].

$$
P D V=\left|\left(R_{X A}\right)-\left(T_{X A}\right)-\left(R_{X B}\right)-\left(T_{X B}\right)\right|=\left|\left(T_{X B}\right)-\left(T_{X A}\right)-\left(R_{X B}\right)-\left(R_{X A}\right)\right|
$$

\subsubsection{Mean opinion score}

MOS value refers the degree of the high-quality excellence of every voice and video telecommunication cellular offerings, the numerical value of MOS shall variety from 1 to 5 ; 1 being the worst quality and 5 being the great high-quality, as proven in (4) a procedure that could be highly timeconsuming Where $\mathrm{R}$ are the individual ratings for a given stimulus by $\mathrm{N}$ subjects [40].

$$
M O S=\frac{\sum_{n=0}^{N} R_{n}}{N}
$$




\subsubsection{Verage throughput}

Throughput is the measure of overall quantity of packets which have efficiently reached the opportunity destination node inside a wireless network. On this context, the numerical value of the throughput shall usually be high, because it directly impacts the overall performance and class of offerings stated within the $4.5 \mathrm{G}$ networks. In this sense, calculate the physical layer information throughput for a few instances, as shown in (5) [40].

$$
\text { Throughput }=\text { Data }[\text { frames }] / \text { Time }[s]
$$

\subsubsection{Signal-to-noise ratio}

The SNR can be described as the ratio among the power strength of the original signal and the power strength for the unwanted noise. Further, it's also used to discover the sensitivity common overall performance of the destination. consequently, the appropriate values of the SNR for alerts shall be above 25 $\mathrm{dB}$ for the multimedia services, as illustrated in (6); where: ' $\mathrm{P}$ ' stands for the strength of the incoming signal; 'I' stands for the interference power of the interfering signals inside the network; and ' $N$ ' stands for some noise terms that could be constant or random [41].

$$
\operatorname{SNR}(x)=P /(I+N)
$$

\subsubsection{Bit error rate}

The BER can be described as the ratio between the overall numbers of errors to the complete quantity of transmitted bits. In this sense, if the channel among the deliver and the destination is at an excellent state with better SNR, the BER is probably very small and will realize the excellent quality and offerings; as a result, the video and voice signals are inside the best case, as described in (7) [42].

$$
\text { BER = Errors } / \text { Total Number of Bits }
$$

\section{SIMULATION, RESULTS AND ANALYSIS}

\subsection{Simulation of scenarios}

In the presented case, three identical scenarios have been implemented in order to differentiate between three IP traffic management schemes MiPV6, IPv6-MPLS and the IPv6-GMPLS schemes based on the LTE-A network. In addition, as shown in Figure 6, one mobile user has been used in the three networks rotating around three eNodes with the mobility feature with a high coverage-rotating radius. The path loss fading effects around three eNodes at the simulation runtime, which equals 482 seconds. In addition, shown in Tables 1, 2 and 3 that the three traffic management scenarios that three types of application used voice, video, and HTTP traffic by using three application servers. The OPNET Modeler 17.5 (Version 8) simulator is used for modeling the three scenarios. Furthermore, critical performance matrices are used to examine the performance of the overall LTE-A network such as (PDV, E2E delay, MOS, Jitter, Traffic Sent Received and the PLR) in the voice, video and HTTP heavy browsing data. Additionally, the BER, SNR, Downlink Packet Drop, Throughput used for measuring the performance of the overall LTE-A network.

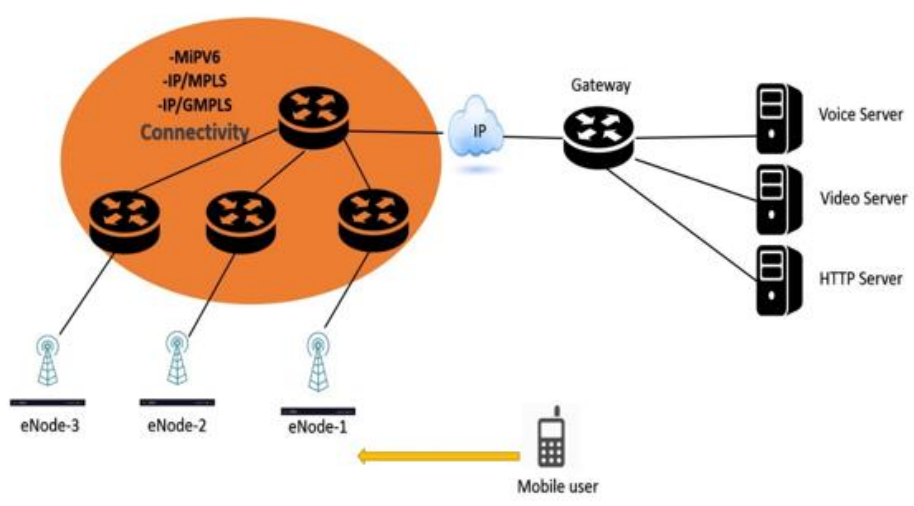

Figure 6. Network topology of the three IP schemes 
Table 1. Voice parameters

\begin{tabular}{ll}
\hline Parameter & Value \\
\hline Application Silence Length (s) & Mean 0.65, Exponentially distributed, \\
De-Compression Delay (s) & 0.03 second \\
Talk Spurt Length (s) & Mean 0.352, Exponentially distributed \\
Encoder Scheme & PCM \\
Type of Service & Best effort $(0)$ \\
\hline
\end{tabular}

Table 2. Video parameters

\begin{tabular}{ll}
\hline Parameter & Value \\
\hline Frame inter-arrival time information & 16 frame/sec \\
Frame size information(bytes) & $128 * 240$ pixels \\
Type of Service & Best effort \\
\hline
\end{tabular}

Table 3. LTE-A network parameters

\begin{tabular}{ll}
\hline Parameters & Value \\
\hline SC-FDMA (UL) Frequency & $1710 \mathrm{MHz}$ \\
Hybrid OFDMA (DL) Frequency & $2110 \mathrm{MHz}$ \\
Transmission Power & $26 \mathrm{dBm}$ \\
Gain Antenna & $17 \mathrm{dBi}$ \\
Antenna Height & $40 \mathrm{~m}$ \\
Radius Coverage & $25 \mathrm{Km}$ \\
Propagation Model & Urban \\
Duration of simulation & $482 \mathrm{~s}$ \\
\hline
\end{tabular}

\subsection{Simulation results}

\subsubsection{Case-1: voice}

As shown in Figure 7, there is an immense variation in the voice quality according to the MOS; thus, using the MiPV6, the value of MOS is about 1.12. In addition, there is degradation up to 2.6 on the quality of voice using IPv6-MPLS then it will increase to 4.2 all over the simulation after the use of the IPv6GMPLS scheme. Therefore, based on the resulting high quality for voice, we conclude that using IPv6GMPLS scheme is preferred. Morever, as illustrated in Figure 8, in case of using the three schemes, the variation of packet delays satisfies lower values that verify 0.000001 second by using IPv6-GMPLS. In case of using the IPV6-MPLS and MiPV6, packet delay variation will differ from 0.0002751 to 0.0008 respectively by using MiPV6.

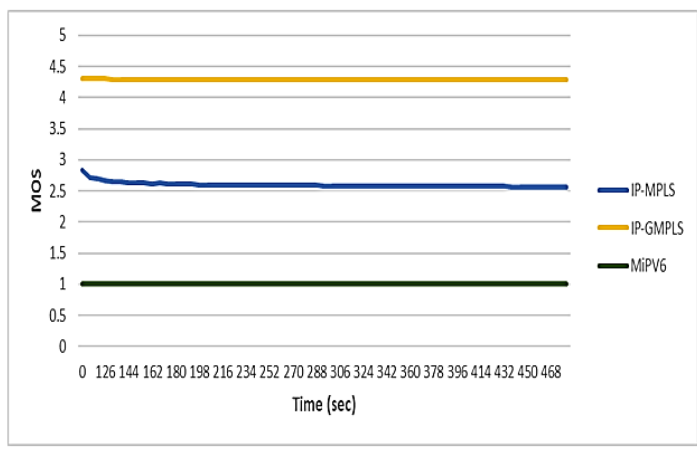

Figure 7. MOS using three IP schemes

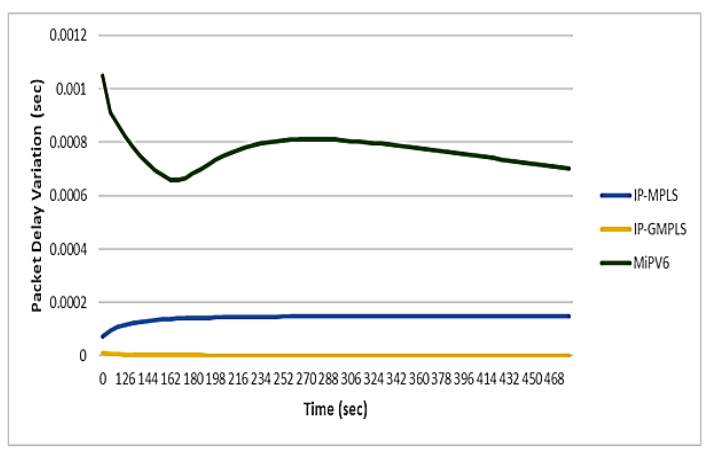

Figure 8. PDV using three IP schemes

According to Figure 9, a small voice jitter value approximately equals zero, after the use of IPv6GMPLS scheme, compared to $-0.00000124 \mathrm{sec}$ with the IPv6-MPLS; then by using the MiPV6 the value will increment up to $0.00002 \mathrm{sec}$. Then will decrease to $0.0000042 \mathrm{sec}$ at the end of simulation this shall in turn influence the overall transmitted voice traffic. In addition, Figure 10 illustrates that the value of the E2E delay satisfies values near to 0.10376 and 0.61034 seconds in case of using the IPv6-GMPLS and IPv6MPLS enabled respectively. On the other hand, it will be equal to 3.278 seconds by using the MiPV6 at the end of the simulation. 


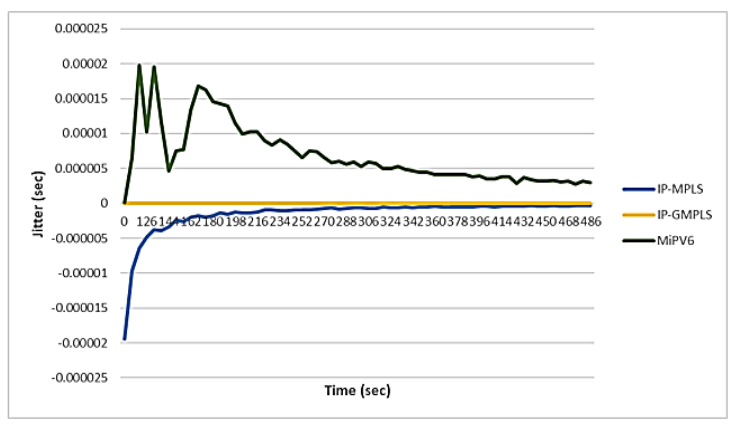

Figure 9. Jitter using three IP schemes

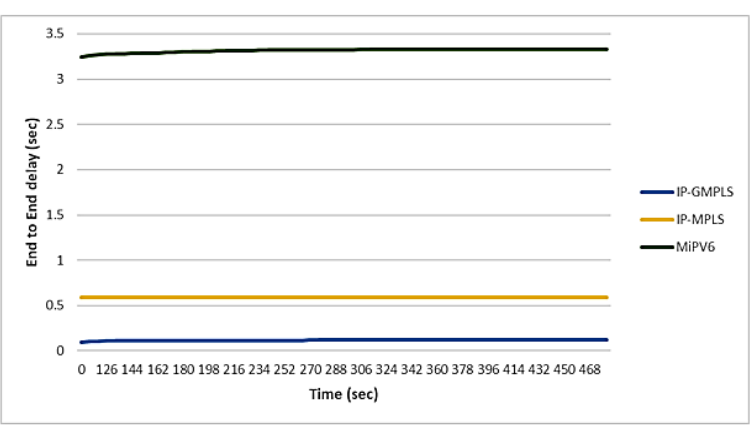

Figure 10. E2E Delay using three IP schemes

Figure 11 shows that the average traffic sent over the three schemes respectively equals 26000 (bytes/sec) at 482 seconds. However, Figure 12 illustrates that the traffic received through the use of IPv6GMPLS approximately equals about 26000 (bytes/sec), and there are no losses with the IPV6-GMPLS. Nonetheless, in case of using MiPV6, there is a big packet drop in the traffic received, thus, approximately greater than $50 \%$ of the voice traffic dropped. On the other hand, approximately $40 \%$ of the traffic dropped using IPV6-MPLS scheme hence, the packet loss ratio can be accumulated for each type, which equals $0.00523 \%, 42.308 \%$, and $57.7 \%$ respectively for the IPv6-GMPLS, IPv6-MPLS and MiPV6 schemes at the end of the simulation.

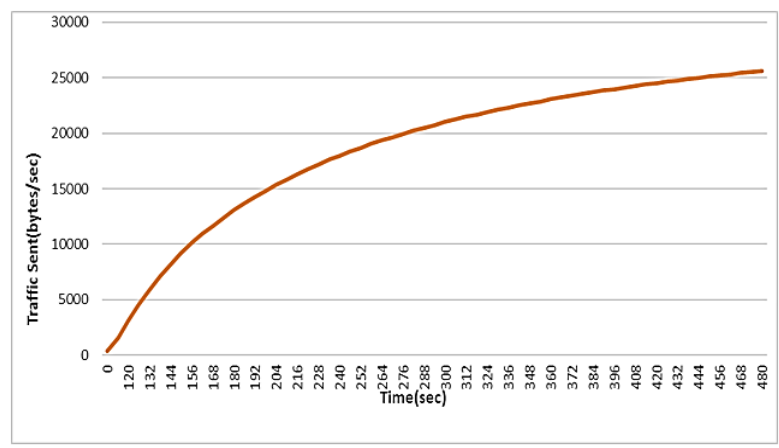

Figure 11. Traffic sent using three IP schemes (bytes/sec)

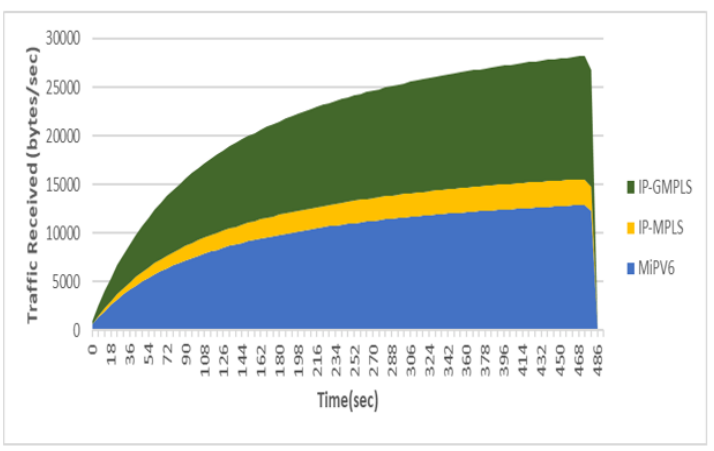

Figure 12. Traffic received using three IP Schemes

\subsubsection{Case-2: video streaming data}

According to Figure 13, the values of the video E2E delay satisfy values after using the three mobile IP schemes, enabled respectively at the end of the simulation. However, the video E2E delay with the MiPV6 has a very high value under the condition of mobility and high coverage area approximately equal 2 seconds, it is a very big value for the delay, which will have a great effect on the quality of received traffic compared to the IPv6-MPLS, which approximately equal 0.9523 seconds. On the other hand, the IPv6-GMPLS is the best end-to-end delay value, which equals 0.000231 second, which is an acceptable value less than $500 \mathrm{msec}$ from the beginning to the end of the simulation time.

As shown in Figure 14, packet variation will be a very high value according to the high coverage area, hence, the accumulated value by using MiPV6 is 0.7102 second, it is a very big value and will effect on the traffic quality. Additionally, IPv6-MPLS packet delay variation will be decremented to 0.3 seconds .om the other hand the IPv6-GMPLS is the lowest packet delay value approximately equal 0.241 at the start of simulation then will be decreased to 0.002451 seconds and that is an acceptable PDV value.

Figure 15 shows that the traffic sent using the three IP mobility schemes over the LTE-A network using approximately equals 520000 (bytes/sec). Illustrated in Figure 16, it will be a big traffic loss using the MiPV6 scheme, which is due to the high values of the E2E delay and packet delay variation. Traffic received by using MiPV6 280000 bytes per second, then will increment by using the two other methods IPv6-MPLS and IPv6-GMPLS, which will equal 519500 and 505340 respectively. Therefore, the metric of PLR accumulated for the IPv6-GMPLS, IPv6-MPLS, and MiPV6 will equal $0.0961 \%$, 2.819\%, and 46.15\% respectively. 


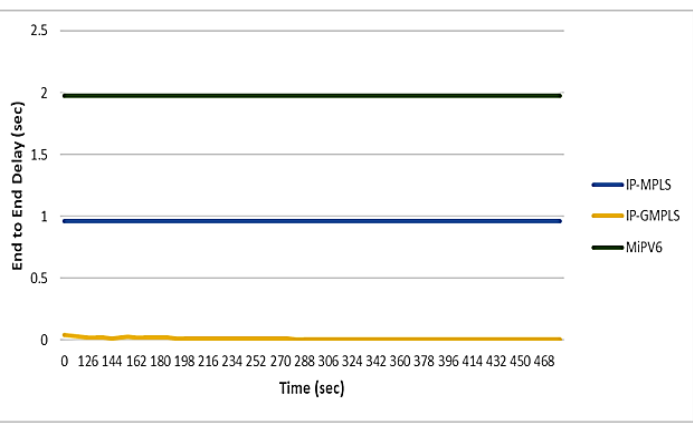

Figure.13 E2E Delay for three IP schemes

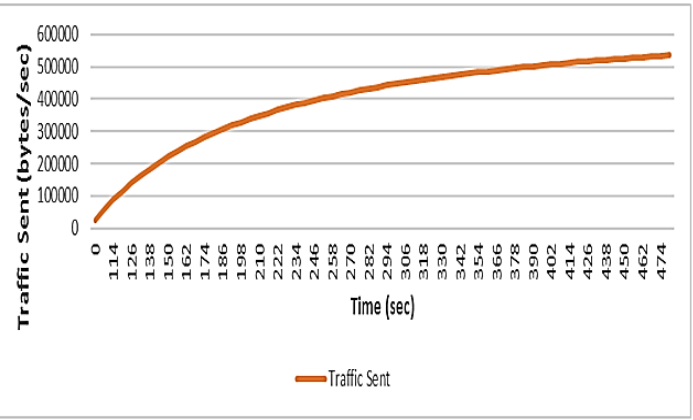

Figure 15. Traffic sent using three IP schemes (bytes/sec)

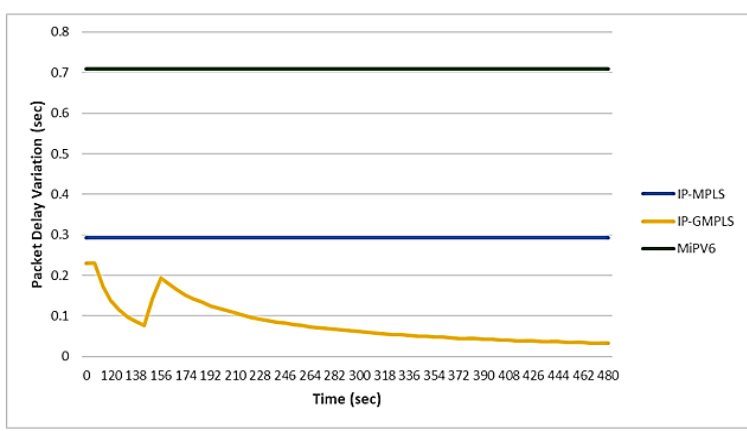

Figure 14. PDV for three IP schemes

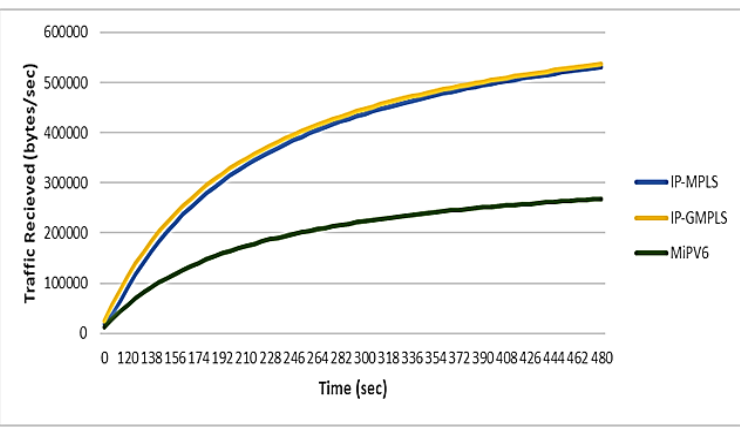

Figure 16. Traffic received using three IP schemes

\subsubsection{Case-3: HTTP}

In Figures 17 and 18 shown that for the HTTP web browsing traffic the MiPV6 satisfied higher delay object and page response values approximately equal 6.9 seconds then increase up to 13 seconds and 0.39 second respectively. This will cause a bad influence directly on the traffic received quality compared with IPv6-MPLS and IPv6-GMPLS respectively these object response time values accumulated to be equal 2 seconds and 0.00012 seconds also the page response time approximately equal 0.05 and 0.01 second respectively. Additionally, In Figures 19 and 20, illustrated HTTP traffic sent at the start of simulation will be 63 bytes sent per every second then will decrement to 15 bytes per second at the end of simulation time, there are huge bytes dropped in the traffic received by using MiPV6 due to high response delay values. On the other hand, by the use of IPv6-MPLS and IPv6-GMPLS bytes received increased to 44 bytes per every second then decreased to 12 bytes per second and 59 bytes per second to 14.5 bytes per second at the end of simulation. The PLR values equal $1.6 \%, 26.6 \%$ and $80 \%$ respectively for IPv6-GMPLS, IPv6-MPLS and MiPV6.

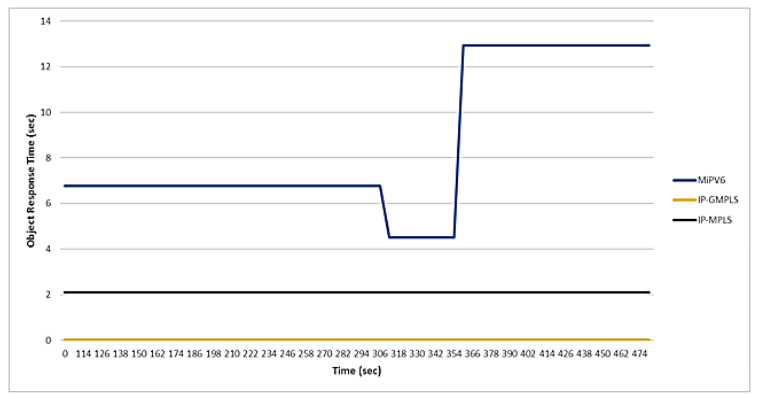

Figure 17. Object response time using three IP schemes (sec)

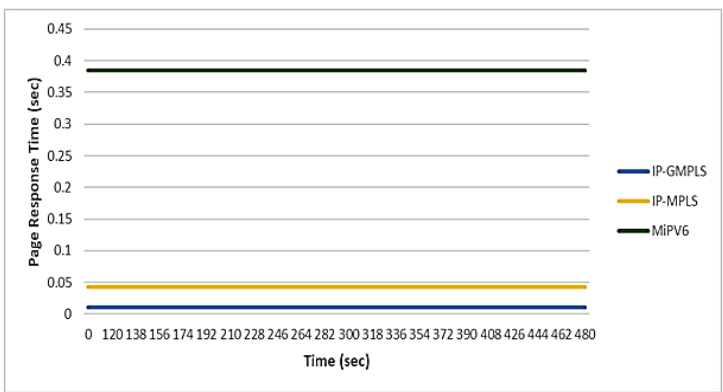

Figure 18. Page response time using three IP schemes (sec) 


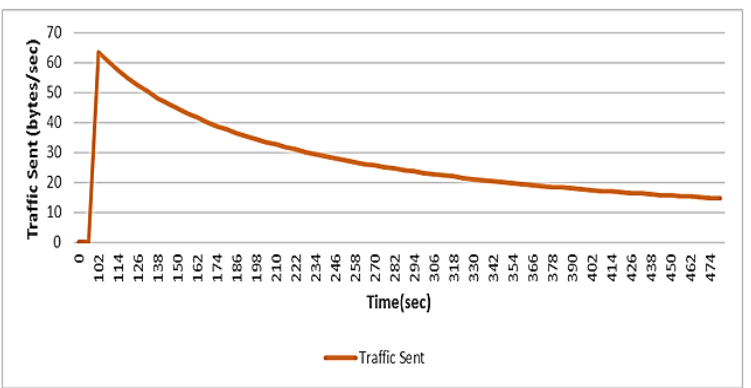

Figure 19. Traffic Sent using three IP schemes

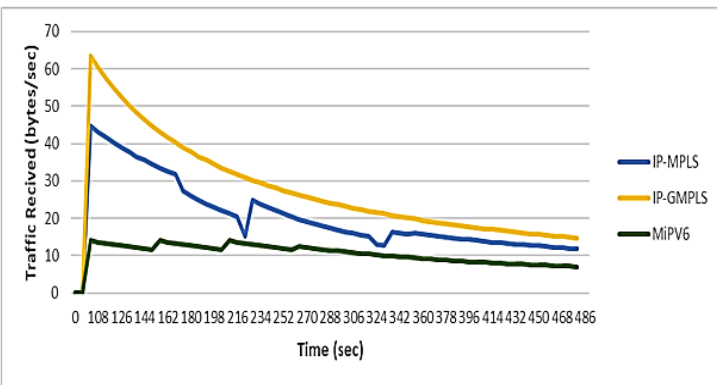

Figure 20. Traffic received of three IP schemes

\subsubsection{Case-4: network performance}

The overall downlink packet dropped over the LTE-A network and by using the three schemes according to Figure 21 approximately $0.1,0.26$ and 0.512 packets per second by using IPv6-GMPLS, IPv6MPLS and MiPV6 respectively. Hence shown there is a huge effect on the multimedia streaming by the use of IPv6-GMPLS on the LTE-A networks. In addition, according to our framework and as shown in Figure 22, the accumulated bit error rate values in case of using the IPv6-GMPLS are very small (about zero) over the downlink which will have an excellent influence on the multimedia streaming over the LTE-A networks compared with the other schemes. According to the simulated system and as shown in Figure 23, with using the IPv6-GMPLS scheme there was a very good throughput value accumulated approximately equal 6000000 bits per seconds at the end of simulation time compared with 4500000 bits per second by using IPv6-MPLS. On the other hand, the worst values accumulated by using MiPV6 which affected the overall multimedia streaming shown in the previous results. Illustrated in Figure 24, all the three schemes satisfy the acceptable values of the signal to noise ratio, the acceptable values of SNR must be larger than 23 $\mathrm{dB}$ in general.

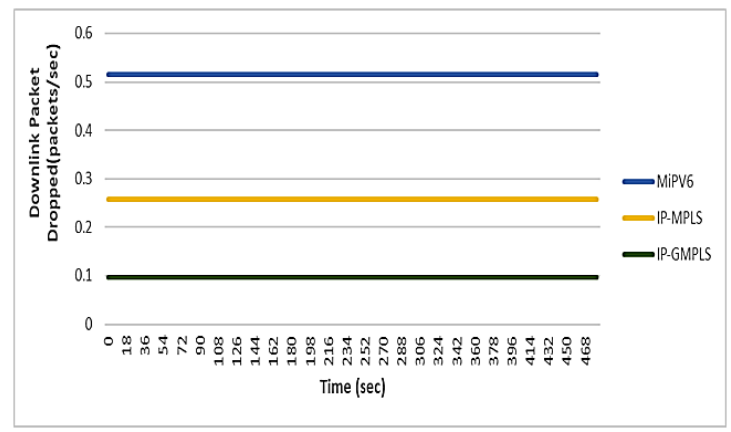

Figure 21. DL Packet drop using three IP schemes

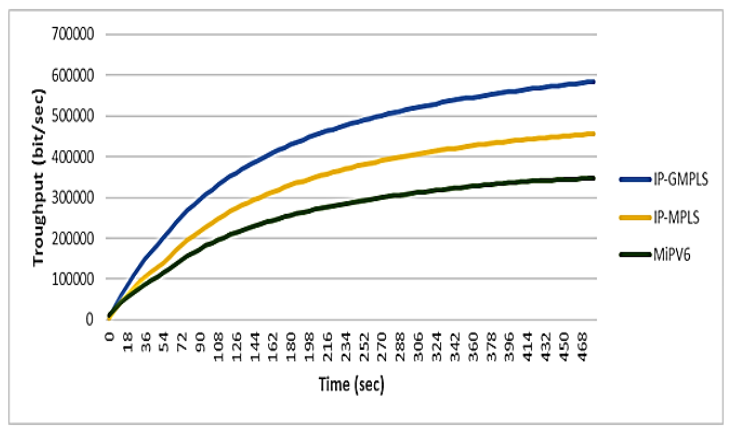

Figure 23. Throughput using three IP schemes

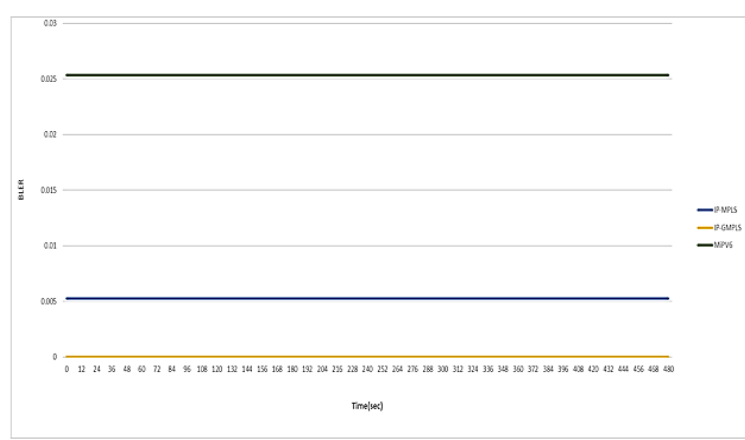

Figure 22. DL BLER using three IP schemes

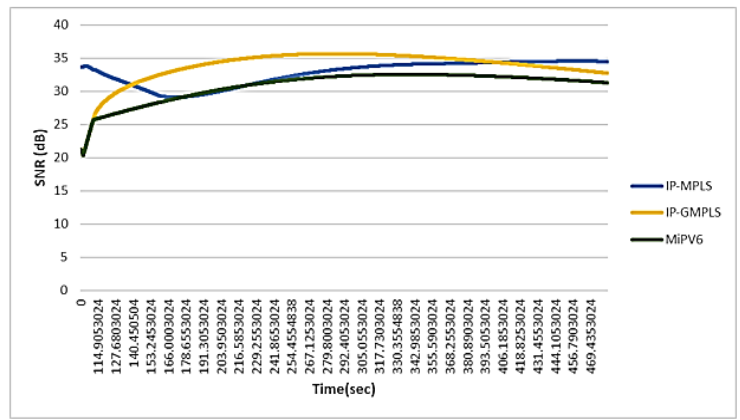

Figure 24. Downlink SNR of three IP schemes 


\subsubsection{General discussion}

In this current case study, three IP traffic management schemes discussed the Mobile IPv6, IPv6MPLS and IPv6-GMPLS, thus a complete comparison was provided between the three types of traffic management proposed schemes to maintain which one is better for the multimedia data streaming and totally LTE-A network performance under conditions of higher coverage, fading effects and mobility. According to results obtained, we found out that IPv6-GMPLS is an excellent choice for transferring heavy loads of traffic such as HTTP browsing, voice and video streaming. Usage of the IPv6-GMPLS in the core routers of the entire LTE-A network has an excellent impact on a critical voice performance matrices such as higher MOS values, lower packet delay variation, lower end-to-end delay, lower jitter, lower packet loss ratio values and higher bytes received compared with MiPV6 and IPv6-MPLS as mentioned in Figure 25, 26. Additionally in case of video streaming, the accumulated results showed a great influence by using IPv6-GMPLS compared with other IP schemes as illustrated in Figure 25, 26. Furthermore, IPv6-GMPLS effected on the quality of HTTP traffic, lower PLR value, lower delays and higher traffic received compared with MiPV6 and IPv6MPLS as stated in Figure 25, 26. On the other hand, we have addressed the influence of the three schemes on the overall performance of the LTE-A network. The accumulated results illustrated that there is a great impact of using IPv6-GMPLS over the LTE-A performance such as higher SNR values, lower BER, lower downlink packets dropped per second and higher throughput bits per every second as shown in Figure 27. All of these obtained results preferred using IPv6-GMPLS in the entire LTE-A network than other methods.

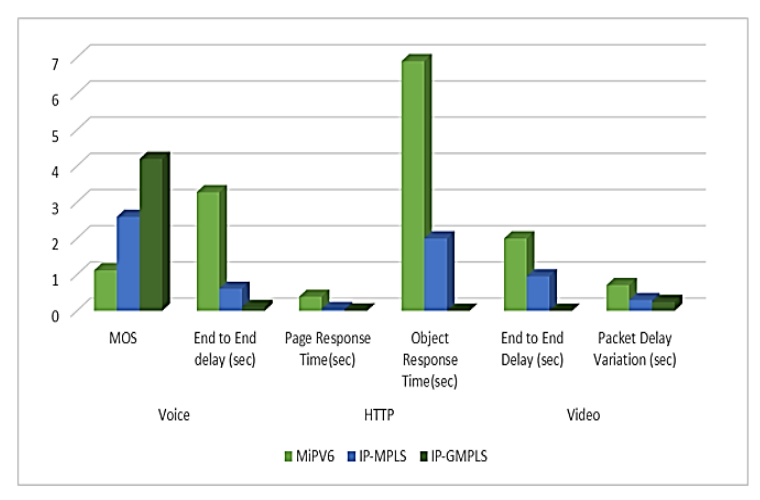

Figure 25. Multimedia results chart

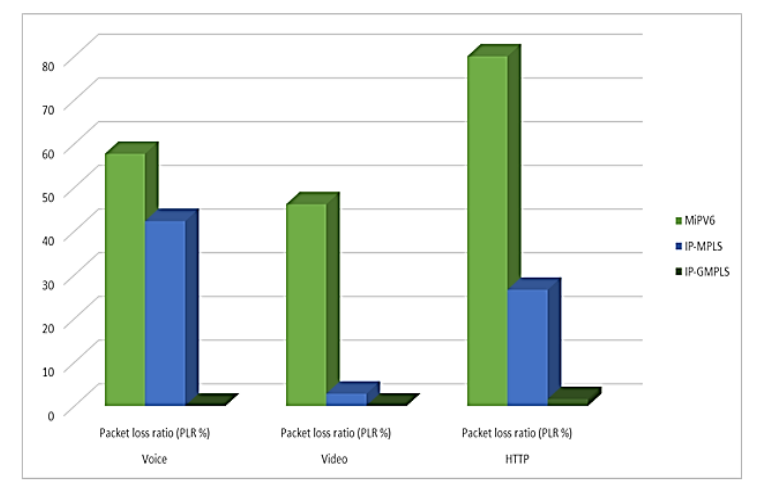

Figure 26. PLR results chart

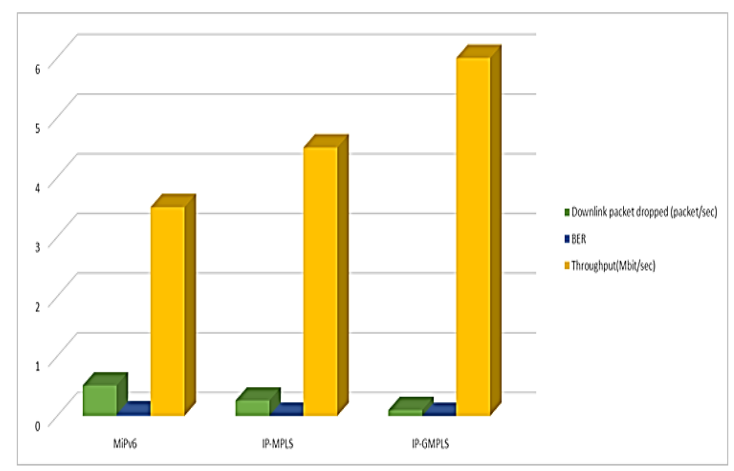

Figure 27. Overall network performance chart

\section{CONCLUSION AND FUTURE REMARKS}

Due to incrementing of the mobile client service needs, with the network use growth and traffic hugeness. Mobile operators be to have the capacity to use new labeling techniques in the core to increase the overall quality of service. In this paper, we have introduced a real-time simulation; and the results showed that the Mobile LTE-A network could deliver sufficient bandwidth, while guaranteeing that the packet delays and jitter will fulfill the required parameters of multimedia streaming. In other words, we have addressed the OPNET simulated networks in order to identify the impact of IP mobility schemes across the LTE-A networks on multimedia streaming, with the objective of accomplishing a good empirical quality value on 
both multimedia and the overall performance. For this end, we have presented an analysis for a number of critical parameters such as the end-to-end delay, packet delay variation, throughput, MOS, SNR, BER and PLR. Thus, after performing the simulation, the obtained results proved that through the use of the IPv6GMPLS scheme, a great influence on both the transmission quality and the overall performance was noticed; and that is in comparison to the other IP schemes. Therefore, in light of this study, we recommend conducting further future studies for more models for smart transmission as well as its effect on data streaming across the mobile networks. Taking into consideration the various types of network connections and the impact of the several fading effects as well as the different conditions of the network shall be studied in detail, with the main objective of providing the best quality at all times of the service's access.

\section{REFERENCES}

[1] R. Zhang, et al., "LTE-unlicensed: the future of spectrum aggregation for cellular networks," IEEE Wireless Communications, 22.3, pp.150-159, 2015.

[2] A. Elakkiya and P. Selvaraj, "QoS based IP mobility management scheme for the next generation SDN-LTE network," In: 2018 second International Conference on Inventive Systems and Control (ICISC). IEEE, pp1355-1360, 2018.

[3] A. Majumder, et al., "Classification of Handoff Schemes in a Wi-Fi-Based Network," In: Enabling Technologies and Architectures for Next-Generation Networking Capabilities, IGI Global, pp. 300-332, 2019.

[4] P. Shah, et al., "A Route Optimized Distributed IP-Based Mobility Management Protocol for Seamless Handoff across Wireless Mesh Networks," Mobile Networks and Applications , 23.4, pp: 752-774, 2018.

[5] R. Meenakshisundaram, et al., "An Integrated IP-MPLS Architecture for Next Generation Networks," International Journal of Computer Applications, 104.3, 2014.

[6] S. Kannan, et al., "Throughput Evaluation of IPV4/IPV6 Networks," 2015.

[7] J. Amutha, et al., "An Integrated Secure Architecture for IPv4/IPv6 Address Translation between IPv4 and IPv6 Networks," In: Proceedings of the Second International Conference on Computer and Communication Technologies, Springer, New Delhi, pp: 669-679, 2016.

[8] Y. A. Amgahd and R. Yadav, "Survey of Mobile IP Protocols," International Journal of Current Engineering and Technology, 6.1, 2016.

[9] W.F.Elsadek and M. Mikhail, "IP mobility management using software defined networking: A review," In: Technology, Networking, Electronic and Automation Control Conference (ITNEC), 2017 IEEE 2nd Information. IEEE, p. 76-81, 2017.

[10] A. Elakkiya and P. Selvaraj, "QoS based IP mobility management scheme for the next generation SDN-LTE network," in: 2018 2nd International Conference on Inventive Systems and Control (ICISC). IEEE, pp: 1355-1360, 2018.

[11] A. Igrair and R. Yadav, "Modified Privacy-Preserving Universal Authentication Protocol (MPriAuth) for Security of Mobile IP Networks," International Journal of Applied Engineering Research, 13.5: 2865-2871, 2018.

[12] S. P. A. Ringland and F. Scahill, "Method and apparatus for a mobile node to connect different access routers while maintaining a consistent network address," U.S. Patent, No 9,961,016, 2018.

[13] K. Pokhrel, et al., "Binding lifetime based signaling cost analysis of multilayer MIPv6," Journal of Computers, 13.3: 337-351, 2018.

[14] H. Anandakumar, et al., "R. A Study on Mobile IPv6 Handover in Cognitive Radio Networks," In: International Conference on Computer Networks and Communication Technologies, Springer, Singaporep, 399-408, 2019.

[15] S. Bryant, et al., "MPLS Flow Identification Considerations," 2018.

[16] I. J. Wijnands, et al., "Encapsulation for Bit Index Explicit Replication (BIER) in MPLS and Non-MPLS Networks," 2018.

[17] A. Bahnasse, et al., "Novel SDN architecture for smart MPLS Traffic Engineering-DiffServ Aware management," Future Generation Computer Systems, 2018, 87: 115-126, 2018.

[18] S. H. Sridhar and J. Arunnehru, "Traffic Engineering an Application of MPLS L3 VPN Technology," In: 2018 second International Conference on Trends in Electronics and Informatics (ICOEI),IEEE, p. 1147-1151, 2018.

[19] H. E. Wahanani, et al., "Performance Analysis Of Video On Demand And Video Streaming On The Network Mpls Traffic Engineering," International Journal, 15.50: 141-148, 2018.

[20] Y. Sinha, et al., "MPLS based hybridization in SDN," In: Software Defined Systems (SDS), 2017 Fourth International Conference on. IEEE, p. 156-161, 2017.

[21] H. O. Otieno, "MPLS (Multi-Protocol Label Switching) assisted routing procedure in Software Defined Networking (SDN)," PhD Thesis, Strathmore University, 2018.

[22] T. Pandikumar and Z. Wondimu, "An Alternative Data Security and Load Balancing in Multiprotocol Label Switching Wireless LAN,” International Journal of Engineering Science, 12327, 2017.

[23] D. Terefenko, "Comparison of Multiprotocol Label Switching (MPLS) and OpenFlow Communication Protocols," 2018.

[24] A. Ghanwani, et al., "Systems and methods for increasing the multiprotocol label switching stack," U.S. Patent, No $9,906,442,2018$. 
[25] M. A. Chishti, et al., "Performance Analysis of Payload Header Suppression (PHS) for Transmission of VoIP over MPLS based IPv4/IPv6 Network," In: Proceedings of the 12th International Conference on Ubiquitous Information Management and Communication. ACM, p. 21, 2018.

[26] K. S. Chen, "Label stacking scenarios in hybrid wavelength and code-switched GMPLS networks," Electronics, 7.10: 251,2018

[27] X. Zhang, et al., "GMPLS OSPF-TE Extensions in Support of Flexi-Grid Dense Wavelength Division Multiplexing (DWDM) Networks," 2018.

[28] R. Rao, et al., "OAM in OTN networks: GMPLS signaling for TCM," U.S. Patent, No 9,253,067, 2016.

[29] J. S. Choi, "Design and implementation of a stateful PCE-based unified control and management framework for carrier-grade MPLS-TP networks," Journal of Lightwave Technology, 34.3: 836-844,2016.

[30] D. Chatterjee, et al., "Signaling for proximity services and D2D discovery in an LTE network," U.S. Patent, No 9,788,186, 2017

[31] P. Gaal, et al., "Expanded search space for R-PDCCH in LTE-A," U.S. Patent, No 9,276,722, 2016.

[32] W. Chen, et al., "Ultra-low latency LTE uplink frame structure," U.S. Patent, No 9,844,072, 2017.

[33] A. Gholmieh, et al., "Streamlined user plane headers for high data rates," U.S. Patent, Application No 15/729, 014, 2018.

[34] G. Nardini, et al., "A scalable data-plane architecture for one-to-one device-to-device communications in LTEAdvanced," Computer Networks, 131: 77-95, 2018.

[35] J. Huang, et al., "Converged network-cloud service composition with end-to-end performance guarantee," IEEE Transactions on Cloud Computing, 6.2: 545-557, 2018.

[36] N. J. Gomes, et al., "Boosting 5G through Ethernet: How Evolved Fronthaul Can Take Next-Generation Mobile to the Next Level,"IEEE Vehicular Technology Magazine, 13.1: 74-84, 2018.

[37] Iskandar, et al., "Inter-cell Interference Management Technique for Multi-Cell LTE-A Network", International Journal of Electrical and Computer Engineering (IJECE), 7(5), 2696, 2017.

[38] I. Angri1, et al., "Exponential MLWDF (EXP-MLWDF) Downlink Scheduling Algorithm Evaluated in LTE for High Mobility and Dense Area Scenario", International Journal of Electrical and Computer Engineering (IJECE), 8(3), 1618, 2018.

[39] C. Ke, et al. , U.S. Patent Application No. 10/122,445,2018.

[40] A. R. Nafchi, et al., "Bit Error Rate (BER) analysis of Discrete Fractional Fourier Transform (DFrFT) based OFDM systems," In: Ubiquitous Computing, Electronics and Mobile Communication Conference (UEMCON), 2017 IEEE 8th Annual. IEEE, p. 485-487, 2017.

[41] B. Clerckx, et al., "Guest Editorial Wireless Transmission of Information and Power-Part I," IEEE Journal on Selected Areas in Communications, 37.1: 1-3, 2019.

[42] P. S. Ravind, et al., "Bit Error Rate (BER) Performance Analysis of DASH7 Protocol in Rayleigh Fading Channel," In: 2018 International Conference on Advances in Computing, Communications and Informatics (ICACCI). IEEE, p. 695-698, 2018.

\section{BIOGRAPHIES OF AUTHORS}

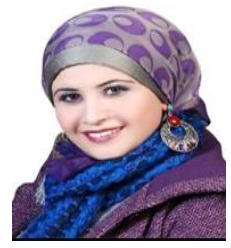

Abeer Twakol Khalil obtained the B.Sc. degree from the Electronics and Communications Engineering Department in 2001. Hence finished the M.sc. degree in 2006 from Mansoura University and the Ph.D. from Mansoura University at 2013. She is now an assistant professor at the Electrical Engineering Department - Faculty of Engineering- Benha University. Also has about 15 published papers in international journals and conferences.

Mail: atwakol213@gmail.com

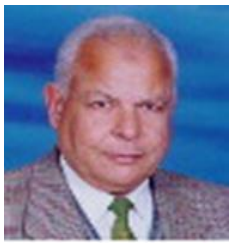

A.I. Abdel-Fatah received his Ph.D. degree from the Department of Electronics and Communications Engineering, Mansoura University, Egypt. He is working now as a professor in the Electronics and Communication Department, Faculty of Engineering, Mansoura University. He has more than 20 published papers in indexed and international peer-reviewed journals in communication networks field.

Mail: abd_fattah@yahoo.com

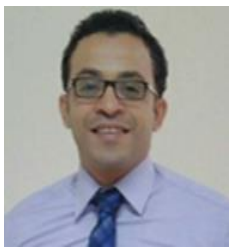

Hesham Ali Sakr received the M.Sc. in Communications and Electronics Engineering from the Faculty of Engineering - Mansoura University - Egypt by 2014 and the B.Sc. degree in Electronics and Communications Engineering from 10th of Ramadan Engineering faculty in 2009. In addition, he has about 5 published papers in international journals. He is working now as a lecturer at Institute of Public Administration - Electronics and Communications department - Saudi Arabia. Mail: Hesham_sakr2010@yahoo.com 